\title{
CONSTRUCCIÓN Y SIGNIFICADO DE LA INTERCULTURALIDAD EN LA ESCUELA NORMAL INDÍGENA INTERCULTURAL BILINGÜE «JACINTO CANEK»
}

\author{
Sergio Iván Navarro Martínez \\ s-navarro@hotmail.com \\ Universidad Intercultural del estado de Tabasco \\ México \\ Antonio Saldívar Moreno \\ asaldivar@ecosur.mx \\ El Colegio de la Frontera Sur \\ Unidad San Cristóbal de Las Casas, Chiapas \\ México
}

\section{RESUMEN}

La relativa reciente aparición del paradigma intercultural ha derivado en que al implementarlo en las distintas experiencias educativas se presenten diferentes problemas. Principalmente el hecho de que se confunde la interculturalidad con aspectos que se reducen al rescate de las tradiciones y elementos culturales o a la incorporación de la lenguas indígenas en los procesos educativos. El presente artículo analiza la forma en que es comprendida la interculturalidad y la educación intercultural en la educación superior. El estudio se realiza en la Escuela Normal Indígena Intercultural Bilingüe «Jacinto Canek», ENIIB, ubicada en San Cristóbal de Las Casas, Chiapas. ${ }^{1}$ El planteamiento metodológico de esta investigación es el enfoque metateórico del interaccionismo simbólico, donde se asume que las prácticas educativas son un escenario donde el sujeto interactúa con otros grupos sociales y son estos procesos de interrelación los que contribuyen de forma decisiva a la configuración de la personalidad del individuo.

Palabras clave: educación intercultural, interaccionismo simbólico, construccionismo, cultura, práctica educativa. 


\section{ABSTRACT}

The relatively recent emergence of the paradigm of interculturality has led to various difficulties in its application to different educational experiences. One of the main problems is the fact that interculturality is confounded with aspects that are reduced to reclaiming cultural traditions and elements or to the inclusion of indigenous languages in education processes. This paper analyzes what is understood by interculturality and intercultural education in the field of higher education. The study was conducted at the «Jacinto Canek» Intercultural Bilingual Indigenous Teacher Training School (Escuela Normal Indígena Intercultural Bilingüe «Jacinto Canek» / ENIIB) located in Zinacantán, Chiapas. The methodology developed for this study is based on the meta-theorical approach of symbolic interactionism. It assumes that educational practices are a scenario where the subject interacts with other social groups and that it is these interrelationship processes that decisively contribute to the shaping of an individual's personality.

Key words: intercultural education, symbolic interactionism, constructionism, culture, educational practice. 


\section{ANTECEDENTES Y ELEMENTOS DEL CONTEXTO}

Los programas de desarrollo y las políticas educativas para las zonas indígenas de México se han caracterizado históricamente por ser integracionistas; es decir, se ha pretendido homogeneizar a la sociedad sin tomar en cuenta las particularidades de cada cultura dando como resultado que el sistema educativo no vea necesario - hasta hace poco y de forma limitada - la incorporación de elementos socioculturales y lingüísticos en el proceso de enseñanza-aprendizaje. Esta situación ha originado una serie de conflictos y contradicciones entre el proceso educativo y los usos, prácticas y costumbres de los pueblos originarios. La educación se presenta entonces como una forma de imposición, en la mayoría de los casos, de los valores y actitudes de la cultura dominante, la occidental (Saldívar y Navarro 2007).

A pesar de que se avanzó en la construcción de la idea de «nación mexicana», también se generaron situaciones de inequidad económica, política y social, que padecen diferentes sectores sociales de la población, tanto rural como urbana, pero histórica y particularmente los indígenas. Estas circunstancias institucionalizan una tendencia hacia la construcción de una identidad uniforme basada además en un modelo de educación indígena precario y segregador, que borra las diferencias entre los pueblos e ignora su diversidad de expectativas en torno a la escuela, con lo cual se agudiza la inequidad (Bertely 1998: 8993).

El paradigma intercultural, recientemente incorporado en los discursos políticos, académicos y educativos, pone en evidencia las formas de dominación que se dan de la cultura occidental sobre las minorías étnicas. López y Küper (1999: 37) señalan que en América Latina «los propios indígenas comenzaron a descubrir que el sistema oficial homogeneizante y monolingüe no satisfacía sus intereses, no contribuía a la reproducción de su propia cultura». Es así que en la década de los setenta algunas comunidades comenzaron a reclamar una educación en su propia lengua, conocida como educación bilingüe, a la que posteriormente se le incorporó el calificativo intercultural, con la 
finalidad de recibir una educación que respondiera a las necesidades de formación de cada contexto sociocultural y considerara la reflexión y modificación de las formas de relación injusta entre las culturas.

De esta manera la educación intercultural bilingüe comenzó a ser adoptada por programas educativos en diferentes países. Por mencionar algunos ejemplos, en Bolivia, Ecuador y Nicaragua se llevaron a cabo en los años 80 importantes campañas de alfabetización de adultos en la propia lengua y se constituyeron en verdaderos antecedentes de lo que hoy es la EIB escolar (López y Küper 1999). No hay que olvidar que estas reformas en materia educativa fueron apoyadas a partir de las recomendaciones hechas por el Convenio 169 de la Organización Internacional del Trabajo, OIT, ratificado en 1990, en el que se determina que la educación de los pueblos originarios debe «responder a sus necesidades particulares, y deberá abarcar su historia, sus conocimientos y técnicas, sus sistemas de valores y todas sus demás aspiraciones sociales, económicas y culturales» (Convenio 169 de la OIT, Artículo 27).

En este contexto, para el caso de México el movimiento 500 años de resistencia indígena, de 1992, postuló el lema: «Nunca más un México sin nosotros». Esta situación influyó en cierto sentido en la modificación del artículo $4^{\circ}$ Constitucional —n él se hace mención de la diversidad cultural y lingüística, sustentada en los pueblos indígenas-, y es a partir de ese momento que se reconoce a México como un país multicultural y plurilingüe, es decir, donde coexisten varias culturas en un mismo territorio. Sin embargo, bajo el modelo multicultural, no se pone de manifiesto la manera inequitativa en que se establecen las relaciones entre las culturas. La interculturalidad propone fundamentalmente nuevas formas de relación que permitan un aprendizaje mutuo y equitativo. De esta forma, se cuestiona el modelo integracionista y homogeneizador de las políticas educativas y de desarrollo en el cual se supone que la lengua y la cultura de los grupos indígenas están entre los factores de retraso económico y social. 
El levantamiento armado del Ejercito Zapatista de Liberación Nacional, EZLN, en 1994, fue otro detonante sobre la importancia de construir un proyecto educativo alternativo que considerara los principios interculturales y respetara la condición de bilingüismo. En los diálogos de San Andrés entre el gobierno federal y el EZLN, se discutió el tema de la educación bilingüe e intercultural. Los representantes de los zapatistas se manifestaron por una educación diferenciada, que respondiera a las características socioculturales de cada contexto y cultura ${ }^{2}$ (Gutiérrez 2006). A raíz de esto se habla en México y particularmente en Chiapas sobre la necesidad de redefinir los modelos educativos implementados en las zonas indígenas.

Como consecuencia de estas demandas en el Programa de Desarrollo Educativo 1995-2000 de la Secretaría de Educación Pública, establece de manera más enfática la necesidad de «lograr una congruencia entre escuela y cultura local», se propone que «en el currículo debe incorporarse saberes y comportamientos propios de cada pueblo, valiosos como norma de relación entre las personas y éstas con el medio natural» (SEP 1996: 76). Es así que se plantea que la educación para los pueblos indígenas se adapte a sus necesidades y condiciones de cultura y lengua, población, organización social, formas de producción y trabajo; asimismo se precisa que la educación para la población no indígena debería de combatir las formas manifiestas y encubiertas de racismo y discriminación.

En la declaración universal de Unesco en el año 2001, se eleva a la diversidad cultural como categoría de «patrimonio común de la humanidad, tan necesaria para el género humano como la diversidad biológica para los organismos vivos» (Unesco 2001). Asimismo, en la Ley General de Derechos lingüísticos de los Pueblos Indígenas —que entró en vigor en 2003 - se reconocen y protegen los derechos lingüísticos, individuales y colectivos, de los pueblos y comunidades indígenas, así como la promoción del uso y desarrollo de las lenguas originarias. 
Oficial e institucionalmente, a partir de 2001 el paradigma intercultural se incorporó al discurso del gobierno mexicano con la creación de la Coordinación General de Educación Intercultural Bilingüe, CGEIB, en la Secretaría de Educación Pública, SEP. Su actividad central es: promover nuevas formas de relación entre las culturas en un marco de diálogo y respeto por la diferencia, para proponer una educación que respete la diversidad lingüística y cultural del país y con el propósito de alcanzar tres objetivos fundamentales: i) mejorar la calidad de la educación básica destinada hacia la población indígena, ii) propiciar la existencia de oportunidades de educación cultural y lingüísticamente pertinentes para los indígenas en todos los niveles educativos, iii) favorecer el desarrollo de una educación intercultural para toda la población (SEP-CGEIB 2004).

La creación de CGEIB, a pesar de ser un paso importante, ha recibido una serie de críticas principalmente de organizaciones de la sociedad civil, una de ellas es que atiende de manera específica a grupos indígenas, cuando la población mestiza es la que requiere de manera más urgente una sensibilización sobre la valoración y comprensión de la diversidad cultural, lingüística y étnica de este país.

Sin embargo, a pesar de que en los postulados de CGEIB se marca claramente que la educación intercultural no es exclusiva para los pueblos indígenas, hay una tendencia en los diferentes espacios educativos a separar lo indígena de lo mestizo, asignándole al primero la categoría intercultural. Situación que pone de manifiesto la incomprensión e inoperatividad de este paradigma en la práctica.

\section{INTERCULTURALIDAD}

El impulso a un proyecto de educación intercultural debe orientarse fundamentalmente a abrir nuevos espacios de comunicación entre los individuos, independientemente de sus diferencias y contribuir a «revertir la tendencia a confinar a las culturas originarias y a mantenerlas como reservas culturales» (Fornet 2002: 154). 
El enfoque intercultural pretende no eliminar diferencias sino aprovecharlas en un proceso de enriquecimiento a partir de los conocimientos de los otros y basado en la interacción. Así, la diferencia se concibe como una virtud que implica respeto y comprensión recíproca entre distintas culturas. Supone una relación entre las culturas en condiciones de igualdad.

Un debate fundamental sobre la educación para los pueblos indígenas está centrado en la toma de posición sobre el punto de partida: el reconocimiento de la multiculturalidad es suficiente para modificar las formas de relación entre los pueblos o es necesario reconocer las condiciones en que se establecen estas formas de interacción: desde nuestra perspectiva por supuesto que no es suficiente sólo el reconocimiento. La interculturalidad cuestiona cómo en una situación de relaciones marcadas por una profunda asimetría se puede establecer un diálogo constructivo, un diálogo que nos enriquezca mutuamente. La pérdida de la capacidad de producción cultural conlleva indudablemente a la asimilación de la cultura dominante sobre las demás culturas.

Tal como señala Schmelckes (2003): «la Interculturalidad es un concepto que quiere ir más allá de la multiculturalidad, es un concepto que busca trascender del reconocimiento de la diversidad a la construcción de nuevas formas de relación». La interculturalidad reconoce que la pérdida de la capacidad de producción cultural y la imposibilidad de la apropiación de los procesos productivos conlleva indudablemente a la invasión de la cultura y economía dominante sobre las demás culturas. Rodrigo (1999) en este mismo sentido afirma que detrás del multiculturalismo puede encontrarse los rastros del nuevo racismo, el racismo sin razas, y de una retórica de la exclusión... uno de los peligros actuales es que el principio de exclusión basado en la diferenciación por la raza, categoría que ha sido ya rechazada por la ciencia, sea reemplazado por el de identidad cultural. Esto nos obliga a tener muy en cuenta los objetivos que se dibujan detrás de las distintas propuestas multiculturalistas. 
Se empezó a hablar de multiculturalismo, que denota la coexistencia de culturas diferentes - una concepción excesivamente estática-, y ahora se habla claramente de educación intercultural —una concepción dinámica—, que expresa más adecuadamente la interpenetración de culturas diferentes. Hace referencia a la necesidad de formar a los ciudadanos para que comprendan la multiplicidad cultural de la sociedad del siglo XXI y desarrollar actitudes favorables a la diversidad de culturas y el incremento de interacción social entre personas y grupos culturalmente distintos (Beltrán 2003).

Para muchos la interculturalidad representa un riesgo, ya que favorecer las formas de interacción entre las culturas puede ser también una forma de desaparición y/o integración «silenciosa» de las culturas minoritarias en la cultura dominante. Esta situación surge de no haber resuelto en primera instancia la superación de las diferencias políticas, sociales y económicas que dificultan un diálogo entre iguales. Un indicador claro también podría ser la producción cultural, es decir, una cultura tiende a desaparecer en la medida en que deja de producir nuevos elementos culturales, por lo que a lo largo del tiempo va asimilando nuevos en detrimento de los propios y por tanto tendiendo a desaparecer.

\section{ANÁLISIS DE LA EXPERIENCIA DE LA ESCUELA NORMAL INDÍGENA INTERCULTURAL BILINGÜE «JACINTO CANEK»}

\section{BREVE RECUENTO HISTÓRICO}

Después del año 1994, la Dirección General de Educación Indígena y los Servicios Educativos para Chiapas asignaron recursos para contratar maestros bilingües egresados del nivel medio superior. El requisito para obtener su plaza era tomar un curso de introducción a la docencia que constaba de tres meses. «No obstante, en 1997 se decidió que no fueran contratados más docentes bajo esa modalidad, porque de ahora en adelante se les asignaría una beca para cursar la licenciatura en la Universidad Pedagógica Nacional, UPN, con el compromiso de que al terminar sus estudios se les asignaría una plaza» (José Jiménez, docente ENIIB, 2007). 
Sin embargo, UPN no tenía suficiente capacidad para atender a los estudiantes, es por eso que surge la idea de crear un proyecto que respondiera a las demandas de formación de docentes para el medio indígena. «Entonces están los jóvenes pero si son trescientos sesenta anual, la UPN no tiene esa capacidad de esa cobertura, mejor crear una institución, no se pensaba en la normal, a lo mejor una universidad indígena y así nos nombran a nosotros para construir el currículum, el Plan de estudio 1997, 1998, 1999, ya casi a final del 99 terminó todo un currículum» (Lucas Ruiz, Docente ENIIB, 2007). Al principio se pensaba en un plan de estudios semiescolarizado.

En el proceso de construir el currículum para la Escuela Normal «Jacinto Canek», se realizaron varias visitas a distintas regiones del estado de Chiapas, principalmente Altos, Selva, Sierra y Norte, con la finalidad de hacer un breve diagnóstico en las comunidades sobre las lenguas maternas, y de analizar junto con ellas la pertinencia de un modelo educativo para estudiantes indígenas. Así es señalado por el docente José Jiménez: «hicimos reuniones en las regiones, de qué opina la gente sobre la formación de los jóvenes, de los maestros; en aquel entonces muchos tenían solamente la secundaria y el bachillerato, y si tienen el bachillerato pues no tienen donde cursar su licenciatura; entonces a través de las opiniones se construyó el currículum». En este sentido, la primera propuesta de currículum tuvo una amplia participación de padres de familia, supervisores, autoridades educativas comunitarias.

A finales de 1999 se presentó el proyecto educativo en la ciudad de México ante la SEP, específicamente con el Director General de Normatividad y Profesiones. El plan de estudios era una construcción propia del equipo de maestros comisionados para realizarlo. Una vez que fuera aprobado se crearía la institución. No obstante, ese plan no lo autorizaron porque estaba fuera de la norma, se consideraba un «,plan de estudios rebelde, zapatista pues era una currícula fuera de lo institucional porque su propósito era diferente, como por ejemplo una formación desde y para el contexto sociocultural de los estudiantes 
indígenas. Ese primer plan de estudios se rechazó por considerarse subversivo y autónomo» (entrevista colectiva a docentes ENIIB, 2006).

A pesar de ello, las autoridades propusieron una alternativa, la cual consistía en hacer una compactación con el Plan de Estudios 1997 de las escuelas normales a escala nacional, agregando aspectos específicos de los contenidos en la propuesta del plan de estudios de ENIIB. «Fue así como aceptaron las condiciones y se nombró al proyecto Licenciatura en Educación Indígena Intercultural Bilingüe, con modalidad escolarizada» (entrevista colectiva a docentes ENIIB, 2006).

Oficialmente la Escuela Normal Indígena Intercultural Bilingüe «Jacinto Canek» ${ }^{3}$ ENIIB comenzó a funcionar el 2 de octubre de 2000 —en instalaciones rentadas de la Escuela Primaria «Jean Piaget» ubicada en la colonia Erasto Urbina, ${ }^{4}$ en San Cristóbal de Las Casas - con dos grupos de treinta estudiantes cada uno. Desde sus inicios la Escuela Normal «Jacinto Canek» se ha enfrentado a diversos problemas, como por ejemplo la falta de instalaciones propias para realizar sus actividades y la renta de una escuela primaria. Esto originó la demanda de un espacio propio ante las autoridades educativas y municipales. Tuvieron que pasar ocho años para que adquirieran instalaciones propias. Es así que desde principios de 2008 estudiantes, maestros y directivos se encuentran ubicados en la comunidad de Zinacantán.

\section{LA EDUCACIÓN INTERCULTURAL COMO PROBLEMA DE INVESTIGACIÓN}

En distintas experiencias de educación intercultural que se han implementado recientemente a nivel superior se han identificado diferentes problemas:

- Se carece de metodologías que vayan más allá de estrategias de rescate cultural.

- Este tipo de educación se considera solamente para los indígenas.

- Hay desvinculación de la escuela con la vida cotidiana de los estudiantes.

- Se parte de conocimientos estructurados y no de conocimientos locales. 
- La utilización de la lengua originaria en el salón de clases no es parte de una estrategia educativa integral.

- Se imagina la educación intercultural como una forma de vida escolar para lograr la armonía entre los grupos de distintas culturas que hablan distintas lenguas.

Además existe una prevalencia de elementos de la educación tradicional, lo que ocasiona incongruencias en la práctica educativa intercultural. Con base en ello, en la presente investigación se planteó el siguiente objetivo: Analizar y comprender el significado construido y otorgado sobre interculturalidad en la Escuela Normal Indígena Intercultural Bilingüe «Jacinto Canek» (ENIIB 2001).

Las preguntas de investigación fueron las siguientes:

- ¿De qué manera se interpreta y construye la interculturalidad y la educación intercultural en la ENIIB?

- ¿Cómo se le asigna significado y práctica a la interculturalidad en la ENIIB «Jacinto Canek»?

- ¿Cómo se aplican los postulados teóricos metodológicos de la interculturalidad en el espacio educativo?

- ¿Qué implicaciones tiene la acción educativa intercultural en el proceso de construcción de identidad para los estudiantes de la ENIIB?

\section{PLANTEAMIENTO METODOLÓGICO DE LA INVESTIGACIÓN}

Este trabajo se basó en una propuesta de tipo cualicuantitativo. La parte cualitativa se aborda desde el enfoque metateórico del interaccionismo simbólico, ya que aporta elementos para comprender cómo se le asigna significado a la interculturalidad. La investigación también se apoya en el enfoque del construccionismo social, ya que «se puede fijar una posición respecto a la concepción que se tiene sobre [la interculturalidad] y la forma en como construimos el conocimiento acerca de ella» (Wiesenfeld 2001: 121). 
El interaccionismo simbólico es un enfoque definido para el estudio de la vida de los grupos humanos y de su comportamiento. Parte de tres premisas:

- Las personas actúan con respecto a las cosas e inclusive frente a las personas sobre la base de los significados que unas y otras tienen para ellas;

- los significados son productos sociales que surgen durante la interacción;

- los actores sociales asignan significados a situaciones, a otras personas, a las cosas y a sí mismos a través de un proceso de interpretación (Blumer 1986: 2).

Desde el punto de vista de los interaccionistas, la gente interpreta estímulos, y esas interpretaciones, sujetas a una continua revisión conforme al acontecer de los eventos, moldean sus acciones. El mismo estímulo físico puede significar cosas diferentes para personas diferentes e incluso para las mismas personas en situaciones diferentes (Hammersley y Atkinson 1994: 4).

Esta investigación centra el peso en la parte cualitativa, por ello para la selección de informantes se empleó el tipo teórico o intencional (Glaser y Strauss 1967, citado en Wiesenfeld 2001), es decir, los actores o informantes se escogen con algún propósito definido, según la concepción que se tenga del problema o de su fundamentación.

Esta investigación tiene como antecedente el proyecto «Retos de la formación de estudiantes indígenas en el nivel superior en San Cristóbal de Las Casas, Chiapas» ${ }^{5}$. Con el cual se obtuvo un acercamiento con directivos y docentes de instituciones educativas de nivel superior. Y fue a partir de esos resultados que se les plateó a los docentes y directivos de la Escuela Normal Indígena Intercultural Bilingüe «Jacinto Canek» la posibilidad de profundizar sobre la formación de los estudiantes bajo el enfoque intercultural. Se realizaron diferentes reuniones de trabajo para presentarles la propuesta de investigación. Una vez presentada y aceptada la propuesta ante la asamblea de docentes y directivos de ENIIB, se comenzó con visitas recurrentes con la intención de observar las distintas 
actividades programadas, a partir del trabajo etnográfico. En particular se llevó a cabo una «observación participante la cual consiste en participar realmente en la vida y actividades de los objetos observados ... compartiendo incluso temporalmente la vida cotidiana del grupo estudiado» (Mucchielli 1996: 224-225).

Con la observación participante se buscaba tener una mirada desde dentro de la problemática, para describir las prácticas educativas tal y como se desarrollan de manera cotidiana dentro y fuera del salón de clases. Algunos elementos que fueron parte del protocolo de observación: el papel del maestro y de los alumnos, uso de material didáctico, uso del espacio en el aula, la aplicación de los criterios metodológicos para las prácticas educativas interculturales, la relación docentes/estudiantes dentro y fuera del aula. Todo ello se registró en un diario de campo, donde se anotaron los comentarios, reflexiones e inquietudes derivadas muchas de ellas de la observación directa dentro y fuera del aula. Otras notas de campo se plasmaron durante la aplicación de las entrevistas.

Se realizó trabajo con cuatro grupos focales a través de talleres participativos, con estudiantes de segundo, cuarto, sexto y octavo semestre de la licenciatura en educación intercultural bilingüe, de ENIIB. «La meta de este procedimiento es la obtención de una síntesis, consenso para la negociación de construcciones más variadas y ricas en información que las previas» (Wiesenfeld 2001).

Fue a partir de este trabajo con los grupos focales que se identifica a informantes clave con los cuales posteriormente se les pidió su colaboración para generar situaciones de diálogo, con el propósito de que pudieran aportar elementos para el desarrollo de la investigación. Además de que los estudiantes colaboraron con las entrevistas, se les acompañó a las escuelas donde realizaban su práctica profesional con la finalidad de conocer la forma en que la llevaban a cabo bajo el enfoque intercultural. Luego de este acompañamiento se les entrevistó a profundidad. 
En lo que se refiere a las entrevistas aplicadas a los docentes fueron seis semiestructuradas. Para ello se dividió entre quienes estuvieron desde el diseño y creación de ENIIB, y entre aquellos que se incorporaron posterior a su fundación. En el siguiente cuadro se presenta el tipo de técnica de recolección de información aplicada para estudiantes, docentes y directivos, así como también el número de ellas.

Cuadro 1. Técnicas de recolección de información

\begin{tabular}{|c|c|c|c|c|}
\hline \multirow{2}{*}{$\begin{array}{c}\text { Sujetos de } \\
\text { investigación }\end{array}$} & \multicolumn{3}{|c|}{ Entrevistas } & \multirow{2}{*}{$\begin{array}{c}\text { Talleres } \\
\text { investigativo } \\
\text { Encuestas }\end{array}$} \\
\hline & Semiestructuradas & A profundidad & Focal & \\
\hline Estudiantes & 11 & 2 & 2 & 52 \\
\hline Docentes & 6 & - & 1 & - \\
\hline Directivos & 1 & - & - & - \\
\hline
\end{tabular}

Fuente: Elaboración propia a partir de la información obtenida en campo.

Se decidió dejar de hacer entrevistas en el momento en que los entrevistados ya no aportaban elementos relevantes para la investigación, es decir, dejan de emerger nuevos datos, lo que se conoce como saturación teórica. Es así que en el proceso de investigación se obtuvieron diversas entradas de información para tener una mirada amplia sobre la construcción y significado de la interculturalidad dentro de ENIIB.

La investigación se realizó en los ciclos escolares enero-julio y agosto-diciembre de 2007. En el semestre enero-julio 2007, los alumnos inscritos en ENIIB se distribuyeron de la siguiente manera: 72 estudiantes tseltales, 39 tsotsiles, 23 choles, 7 tojolabales, 6 zoques, 4 mam, 3 cachiquel y un kanjobal. Dando un total de 156 alumnos, de los cuales 86 son hombres y 70 mujeres. Es importante señalar que hasta el año 2006 han egresado tres generaciones, la primera fue de 2000 a 2004 con 54 estudiantes, la segunda de 2001 a 2005 con 44 egresados, y la de 2002 a 2006 con 51 egresados.

El área de estudio comprende la ciudad de San Cristóbal de Las Casas, Chiapas y su entorno regional. Se trata de un centro estratégico en Los Altos de Chiapas, en el cual 
confluyen una diversidad de grupos indígenas para realizar diferentes actividades: económicas, sociales, culturales y administrativas; además, como resultado de procesos migratorios, su presencia en la ciudad se ha incrementado significativamente. De esta manera, como parte de este proceso, muchos de los jóvenes indígenas que logran terminar su bachillerato encuentran la posibilidad de continuar sus estudios en alguna institución de educación superior. ${ }^{6}$

Los estudiantes que ingresan a la ENIIB «Jacinto Canek», lo hacen con la aspiración de conseguir posteriormente una plaza como maestros. Quienes egresan trabajan generalmente en contextos indígenas.

\section{SIGNIFICADO Y CONSTRUCCIÓN DE LA INTERCULTURALIDAD}

Como se ha señalado, la interculturalidad es un paradigma que está en construcción, y por ello también puede ser interpretado de diferentes maneras. En este apartado se analiza y reflexiona sobre los distintos significados otorgados a la interculturalidad por los sujetos vinculados al proceso educativo en la Normal «Jacinto Canek».

Se presentan los resultados en las siguientes categorías de análisis: interculturalidad, identidad indígena, educación intercultural. Primero se aborda desde los alumnos y posteriormente con maestros y directivos.

Estos resultados son producto de la sistematización de la información obtenida a partir de la implementación de las estrategias de investigación realizadas con los y las estudiantes, docentes y directivos de ENIIB, y se indaga sobre el significado y construcción de la interculturalidad. Cabe recordar que por significado se entiende de acuerdo con Blumer (1986) a los productos sociales que surgen durante la interacción social. Es así que la primera categoría sobre la cual nos ocuparemos es interculturalidad. 


\section{INTERCULTURALIDAD}

En las entrevistas a los estudiantes, mencionan: «[la interculturalidad es] convivir con otras personas hablantes de [diferentes] lenguas, y conocer las costumbres y tradiciones de cada una de ellas, porque no podemos interactuar con ellas sin conocerlas primero» (Olivia, 20 años, originaria de Huixtán). Asimismo se argumenta que «...la interculturalidad es el intercambio pues, de culturas, por ejemplo dentro del salón de clases nosotros hablamos ocho lenguas, y dentro de ella, está inmerso pues, lo que es nuestra lengua, nuestras costumbres, entonces intercultural es relacionar pues nuestra cultura con otro ... y es intercambio de culturas, de una comunidad y de otros dialectos, entonces yo creo que es variantes como lo podamos llamar, eso es intercultural» (Isael Pérez, 19 años, originario de Pueblo Nuevo).

Este planteamiento se refuerza cuando los estudiantes señalan que «...en la interculturalidad hay muchas culturas en donde se integran uno mismo, en donde se respetan, se conocen» (Agustín, 21 años, originario de Palenque). «[Es] pues la armonía entre las distintas culturas en el mismo espacio» (Edgar, 20 años, originario de la ranchería la Gloria). «En donde llegan a encontrarse en diferentes etnias, y diferentes culturas, entre ellas existen muchas culturas, en el momento cuando ya existen esas, muchas culturas llámese tojolabal, mam, kakchikel, tsotsil, tseltal, entre ellos habría la comunicación, la relación y la convivencia, el intercambio de la cultura, pues» (Carmen, 19 años, originario de Muytajom).

Hay que recordar que si bien es cierto la interculturalidad tiene como elemento importante la interacción social en contextos de diversidad, así como el diálogo y la aceptación gustosa del otro, esta se debe dar bajo condiciones de equidad. Lo que se menciona en el fragmento anterior de la entrevista reduce la interculturalidad a encuentros entre culturas o al hecho de hablar varias lenguas. Particularmente resalta el hecho de que las relaciones planteadas son entre grupos que pertenecen a la cultura maya y no se menciona la relación con la cultura mestiza, donde se genera principalmente el conflicto de interacción inequitativa. La interculturalidad no existe —en tanto las formas de relación entre los pueblos no sean 
equitativas, sino de dominación e imposición-, esto implica implementar una serie de medidas que posibiliten acercarnos a ella (Rodrigo 1999, Gashé 2002, Schmelkes 2001, Fornet 2002). Se parte de que la interculturalidad está en construcción, entonces lo que nos posibilitaría acercarnos a ella es a través de generar las condiciones para lograr un diálogo constructivo y de aprendizaje mutuo (SEP-CGEIB 2004, Saldívar 2003).

En este sentido, la interculturalidad no debe quedar reducida a las relaciones establecidas a partir del uso de las lenguas indígenas en el marco del proceso educativo, a pesar de que estas son una posibilidad de relacionarse de una mejor manera entre las culturas. En el caso de ENIIB debe retroalimentarse de la diversidad cultural y lingüística así como también de las diferencias individuales. Al respecto el Maestro Manuel Bolom señala:

\begin{abstract}
Debemos manejar bien la lengua materna y el castellano. Lo otro desde mi punto de vista es que no debe ser nada más como estamos sectorizando puros indígenas, para mí como fortalecimiento intercultural deben haber un gringo y mestizos, mínimo un gringo digamos, dos o tres mestizos y los indígenas, pues, entonces podamos convivir con ellos o [al contrario puede que sean] ellos [quienes] comiencen a tomar los liderazgo por nuestros referentes culturales que tenemos, si no son gringos que sean alguien que le guste la escuela y pues también nuestros compañeros mestizos ... La interculturalidad es la diversidad, el respeto a la diversidad (Manuel Bolom, docente de ENIIB, 2007).
\end{abstract}

Los docentes también identifican el bilingüismo como un aspecto importante dentro del proceso educativo. En la entrevista anterior también se observa la preocupación por incorporar grupos culturalmente distintos a los indígenas. Pero de nuevo es importante reiterar que el hecho de estar en contextos de diversidad cultural no significa por sí mismo que la interculturalidad se esté llevando a cabo, es necesario modificar las formas injustas en que se establecen las relaciones culturales.

...tal vez cuando escuchamos por primera vez, al entenderlo nos causa mucho problema, nos causa conflictos cognitivo porque ¿qué es la interculturalidad? Yo soy tojolabal, y cuando surge un nuevo concepto, que mira tienes que respetar el otro, que somos igual, que el contacto con la diferencia 
ayuda a ser mejores competentes, pero no es así. Entonces pues necesitamos generar condiciones que nos permite avanzar en un posible reencuentro, tal vez por la primera vez que sea utilizado ese concepto intercultural empezamos a sufrir. Porque ya está el otro en nuestros corazones, entonces al momento de quererlo que esté abajo que ya casi lo estamos exterminando entonces es difícil, es cuando empezamos a sufrir (entrevista colectiva a docentes, ENIIB, 2006).

Es importante señalar que para autores como Fornet (2002) la interculturalidad se nutre de cada contexto y circunstancia que la definen, por lo que no se puede partir de una definición ya elaborada y terminada. En este sentido, se puede hablar de un significado propio y apropiado para la educación intercultural en la Normal «Jacinto Canek», el cual es compartido y retroalimentado en la interacción social entre docentes y estudiantes. Así mismo, Zemelman (1990) menciona que la interculturalidad también se refiere a la capacidad de los sujetos sociales para elaborar, impulsar y sostener proyectos de sociedad; estos últimos resultado de su construcción como fuerzas sociales, como expresión de opciones ideológicas y políticas enraizadas en su cultura.

En este sentido la interculturalidad en la educación superior — en este caso en ENIIB - es un espacio abierto a una parte de la sociedad que por décadas no había tenido cabida dentro de un proyecto de nación. El aprendizaje e intercambio cultural se centra en lo indígena. Señala una estudiante que: «nosotros aceptemos a los demás, tal y como son, aceptar su pensamiento y todo lo que él sabe, y la intercultural más que nada se trata de intercambiar nuestras ideas de nosotros, pues. Por ejemplo en lo que es los mitos para los tsotsiles, hacen rezos en las cuevas o donde nace el agua, igual los tseltales, entonces hay una coincidencia ahí también, y hay otras diferencias ahí también, entonces ahí podemos confrontar y también intercambiar los conocimientos» (Merle, 20 años, originaria de Ocosingo).

Como se observa, hay un distanciamiento con los grupos culturalmente distintos a los originarios, como los mestizos, quienes no tienen presencia en el proceso educativo de ENIIB. «Ese es el conflicto [que] siempre se ha discutido ... la lengua es el puente principal para que el maestro se comunique con los niños, hay comunidades que es 
monolingüe de su lengua materna y si llega un maestro que no habla la lengua es como si fuera una escuela monolingüe, es un problema serio. Entonces en la normal no importa quien llegue o quiera estudiar, lo importante que hable una lengua indígena ese es uno de los requisitos fundamentales para ingresar» (Antonio Girón, directivo de ENIIB, 2007).

A pesar de que hay una apertura para que ingresen estudiantes que no sean indígenas - pero dominen una lengua indígena —, no hay registro alguno que indique su interés por estudiar ahí. Lo cual hasta cierto punto es entendible por los requisitos de ingreso, ya que es obligatorio aprobar el examen de bilingüismo. Es por ello que los estudiantes que ingresan tienen dos rasgos fundamentales: a) son originarios de los pueblos indígenas y b) hablan una lengua materna. Sobre estos dos elementos se construye el significado de la interculturalidad en la Normal «Jacinto Canek», que como se ha observado se comparte entre estudiantes, docentes y directivos.

Es importante resaltar la necesidad de considerar a los otros grupos socioculturales —como mestizos - diferentes a los indígenas, sobre los que hay que incidir e incluir en el proyecto educativo, y no excluir, ya que de lo contrario se corre el riesgo de cometer errores del pasado; es decir, tener una escuela solo para los indígenas, lo cual es contradictorio no solo con el discurso de CGEIB — que habla de una educación intercultural para todos-, sino también con el concepto mismo, ya que la interculturalidad nos permite redimensionar las formas de relacionarnos entre las culturas.

\section{BILINGÜISMO}

En lo referente al bilingüismo, se considera como parte importante en el proceso educativo y también en las formas de relacionarse con los demás, y es que de acuerdo con su Plan de Estudios 2001: «el bilingüismo favorece la adquisición, desarrollo, fortalecimiento y consolidación de la lengua originaria y el español, elimina la imposición de una lengua sobre otra; esto implica que ambas sean consideradas en el proceso educativo como objeto de estudio, medio de enseñanza y de comunicación» (ENIIB 2001: 23). 
En el discurso tanto del plan de estudios como en los mismos estudiantes de ENIIB se reconoce en el bilingüismo una oportunidad para aprender y desarrollar competencias que les permita desenvolverse en un contexto igual o diferente al suyo. «[Y es que] dentro de los rasgos deseables del nuevo maestro se establece que precisamente el estudiante al egresar de la Normal hable, lea y escriba en su lengua indígena» (Lucas Ruiz, docente ENIIB, 2007).

No obstante, la formación que reciben los estudiantes solamente refuerza su lengua materna, sin que les permita aprender otras lenguas. Esto hasta cierto punto es contradictorio, debido a que al egresar deben tener las competencias para laborar en cualquier contexto sociocultural. Uno de los problemas estructurales de la educación en Chiapas es que muchos de los maestros son enviados a zonas que no corresponden a su lengua materna y no están preparados para enfrentar esta situación.

Cabe señalar que aunque el bilingüismo es una parte substancial de la interculturalidad, no debe considerarse como un sinónimo o como su totalidad, es decir, la adquisición de una segunda lengua es una competencia que permite redimensionarse desde un contexto hablante de una lengua originaria. Sin embargo, como se ha visto hasta ahora, la construcción que se hace de la interculturalidad en ENIIB considera al bilingüismo como fundamental para su comprensión y puesta en práctica.

Como se observó en diferentes espacios y en actividades realizadas por la ENIIB, es evidente que el uso de la lengua materna en la mayoría de las ocasiones se da entre hablantes de la misma, y a pesar de que reconocen en ella un aspecto fundamental para llevar a cabo la educación intercultural dentro de aula su uso es muy limitado, predominando el uso del español. 


\section{IDENTIDAD INDÍGENA}

Los estudiantes indígenas, a lo largo de su formación educativa en preescolar, primaria, secundaria y el bachillerato, se encuentran inmersos en un modelo educativo que de forma limitada reconoce como importantes los elementos propios de su cultura. Esto trae consigo una condición permanente de negación cultural que deriva en la adopción de valores y actitudes de la cultura dominante: la occidental.

Un aspecto que se pudo observar durante el trabajo de investigación en la Normal es que la incorporación de estudiantes indígenas en el proceso educativo de ENIIB ha posibilitado de manera importante construir un espacio abierto para las distintas manifestaciones socioculturales, posibilitando la revaloración de su lengua. De igual forma, representa una oportunidad para que los estudiantes puedan re-conocerse como miembros de un grupo étnico determinado. Es así que una de las categorías de análisis es precisamente la identidad indígena, y para indagar sobre la atribución que le asignan los estudiantes, se profundiza sobre el antes y después de ingresar a ENIIB. Con esto se identifica la recuperación y valoración como indígenas. Los siguientes fragmentos de entrevista lo evidencian:

S: ¿Cuál es tu lengua materna?

$\mathrm{V}$ : Es tseltal

S: ¿Siempre la has hablado?

$\mathrm{V}$ : Yo le podría decir que cuando estaba yo en $\mathrm{COBACH}$ [bachillerato] pues nunca hablé mi lengua materna

S: ¿Por qué?

$\mathrm{V}$ : Pues, por lo mismo también de que mis compañeros no hablaban

S: ¿Tenías compañeros ahí que también la hablaban?

V: Sí, tenía yo compañeros indígenas de la misma lengua

S: ¿Pero nadie sabía que la hablaban?

V: No, nadie sabía, ni les dije que yo hablaba tseltal por lo mismo de que tenía yo miedo de que ellos me discriminaran

S:¿Quiénes? 
V: Mis compañeros

S: ¿Por qué cuando hablabas alguna lengua indígena ellos te discriminaban o por qué no te atrevías hablar tu lengua indígena?

V: Pues por lo mismo que he escuchado lo que es la discriminación y que, este, que los mestizos tratan muy diferente a los indígenas, por eso, por lo mismo no quise hablar, pues, mi lengua materna (Víctor, 19 años, originario de Jochib, Oxchuc).

En los medios de comunicación no están claramente representadas las distintas culturas que conforman el país; de igual forma, las diferentes instituciones educativas y aquellas que trabajan en programas de desarrollo en las comunidades indígenas no consideran los elementos culturales como base para la implementación de sus estrategias. En este sentido, existe una situación de negación sistemática de las culturas originarias y de sus lenguas.

En la entrevista se revela que a pesar de tener compañeros con los cuales compartía la misma lengua no se atrevía a hablarla por temor a ser discriminado. Lo cual se convierte en una percepción generalizada entre algunos indígenas. De ahí la necesidad de aprender el español como un mecanismo de visibilización y como posibilidad para participar en la vida social y económica de la región. Así lo señala una estudiante: «Yo tenía otra idea diferente, de que mi lengua era menos, o sea, incluso pensaba yo dejarlo porque hasta bueno mis papás me decían de que aprenda el español, porque aprendes o sea a la fuerza, pues yo desde la secundaria me mandaron pues en la ciudad, porque ellos pues querían que aprendiera yo español, ya fue que o sea nos dio pues esa idea de que mi lengua o sea es menos, hasta me daba pues pena hablar» (Janet, 20 años, originaria de San Miguel, Salto de Agua).

Stavenhagen (2000) señala que con la expansión de las relaciones de producción capitalistas el indígena se da cuenta de que su propia lengua y cultura no le sirven, lo que constituye incluso un obstáculo para «incorporarse» al Estado nación. Asimismo DíazCouder (1998) indica que hay un combate constante contra la pobreza de los pueblos 
indígenas, debido a que se asocia el «ser indio» con el «ser pobre», y que por lo tanto el dejar de ser pobre es dejar de ser indio. De ahí que generalmente se tenga de manera inconciente en la sociedad una situación abierta y silenciosa de discriminación social y cultural hacia los indígenas.

Es evidente que aún falta mucho por hacer en la cuestión intercultural, a pesar de que se proponga como eje trasversal para la educación pública del país. Es necesario analizar lo que sucede en otros estamentos — como la familia, las instituciones y los medios de comunicación- en los cuales está presente la discriminación y el racismo.

Un aspecto importante es que la identidad indígena de los estudiantes se resignifica una vez que ingresan a ENIIB. Su propuesta educativa establecida en el plan de estudios y en las estrategias pedagógicas tiene la finalidad de que los estudiantes se reconozcan como indígenas. Así es señalado por los docentes:

\footnotetext{
Muchos chavos ya salen concientes de su propia cultura, de su propia lengua convencidos pues, ya nos les da pena porque varios chavos me dicen maestro yo me negué, yo decía que no era indígena cuando estaba en la prepa, en la secundaria, pero ya después me fui dando cuenta que no estaba bien ... pero después de los cuatro años ya salen más convencidos, más concientes, como que aceptan su identidad como indígena (Manuel Bolom, docente ENIIB, 2007).
}

Asimismo se señala que: «Aquí se despiertan, aquí vienen a adquirir conciencia y ya escucho que hablan en su lengua, ya paso lista en su lengua ... aquí ha habido mucha baja autoestima por la discriminación, pero con las conferencias que se hacen con la asignatura de Introducción a la diversidad cultural, y nosotros mismos que estamos detrás de ellos, así es como ellos ahora ya son indígenas ... los programas formativos están orientados hacia la interculturalidad» (Cristina Gómez, docente de ENIIB, 2007). 
Para identificar los cambios que se han dado en los estudiantes a partir de su ingreso en ENIIB, se les preguntó si la interculturalidad les ha cambiado la vida como persona y la forma en que se relacionaban con los demás. Al respecto mencionaron lo siguiente:

\footnotetext{
Ahora más que nada ha cambiado mi actitud, porque a veces hay señoras que llegaban a mi casa, así indígenas, y a veces yo no hablaba sabiendo que sí hablaba la lengua indígena, yo no les contestaba en lengua indígena les contestaba en español y ahora ya no me da vergüenza, hablo con mis tías, con mis papás o en la calle (Merle, 20 años, originaria de Ocosingo).
}

Otra estudiante cuenta que: «Cuando entré en la escuela era totalmente monolingüe, al igual que en la secundaria, en la prepa, y ya hasta ahora hay un cambio totalmente diferente porque me encuentro con personas iguales a mí ... me siento un poco raro al hablar mi lengua porque no la había hablado, pero siento que es importante valorar todos esos conocimientos de nuestros papás, de nuestras comunidades, pues. Aquí he aprendido que no tiene nada malo [hablar la lengua materna], me ha ayudado a aceptar más a mi cultura, a mi comunidad» (Edgar, Ranchería La Gloria, municipio de Ocosingo).

Giménez (2002) menciona que la valoración del propio lenguaje no solo es un medio de comunicación intergrupal, sino también un archivo vivo de su visión del mundo y símbolo distintivo de su identidad social. La lengua aparece entonces como un componente esencial en la construcción de la identidad de los estudiantes. A pesar de esto, es importante señalar que durante los últimos años la relación entre lengua y construcción de identidad étnica empieza a modificarse. Particularmente en los censos de población, se identifican cada vez más personas que sin hablar alguna lengua originaria se reivindican como indígenas.

Díaz-Couder (1998) dice que generalmente se piensa que al atender las particularidades lingüísticas de una comunidad se atienden las particularidades culturales, y por consiguiente se refuerza la identidad étnica. Sin embargo, la identidad social —étnica - de algunos grupos no coincide con la variación cultural observable. Es decir, las categorías lingüísticas y culturales establecidas por los analistas sociales no siempre son significativas 
para la autopercepción de los individuos como pertenecientes a un grupo social determinado.

En este sentido, para Barth (1976) la etnicidad es un producto del proceso de la identificación, que puede definirse como la organización social de la diferencia cultural. Lo que realmente importa para explicar la etnicidad no es tanto el contenido cultural de identidad, sino los mecanismos de interacción que utilizan cierto repertorio cultural de manera estratégica, selectiva, y mantienen o cuestionan las fronteras colectivas. De esta manera, con la organización e interacción social en un espacio determinado — en este caso, la misma escuela — da como resultado la construcción de la identidad —étnica— de los estudiantes que ingresan a ENIIB.

En el siguiente gráfico se aprecia la valoración realizada por estudiantes respecto a los datos obtenidos sobre la percepción de su cultura e identidad.

Gráfica 1. Cultura e identidad

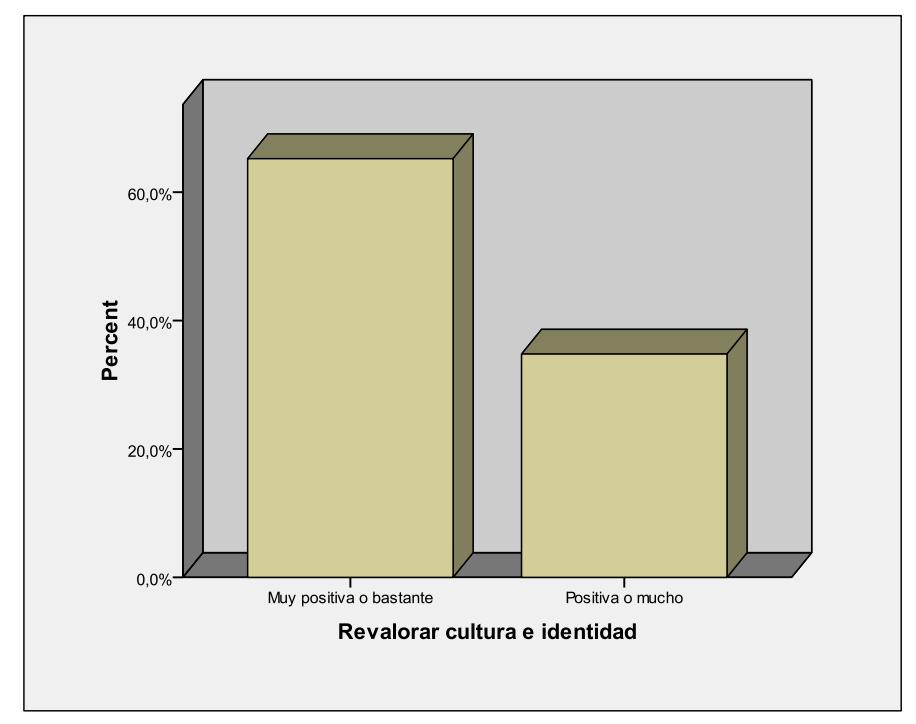

Fuente: elaborada a partir de los resultados de los grupos focales, 2007. 
Lo que se puede observar del gráfico anterior es que hay una valoración muy positiva (65.2\%) sobre la forma en que la educación recibida en ENIIB les ha ayudado a revalorar su cultura e identidad.

A pesar de que en las diferentes actividades realizadas dentro de la Escuela Normal presentan el propósito de que los estudiantes tengan un reencuentro con su pasado indígena $-\mathrm{y}$ esto les permita resignificarse como portadores de una cultura - se ha de reconocer que sigue habiendo una valoración muy fuerte de los elementos de la cultura occidental. Durante las observaciones realizadas en algunos de los eventos culturales organizados por ENIIB, por ejemplo en la conmemoración del aniversario de su fundación, se organizaron diferentes actividades, entre ellas un concurso de canto para los estudiantes, en el que todas las canciones interpretadas fueron en español, ninguna en lengua originaria, tampoco se mostró ningún elemento cultural propio por parte de ellos.

\section{EDUCACIÓN INTERCULTURAL}

En lo referente al trabajo con grupos focales, ${ }^{7}$ se pudo recabar la siguiente información al comparar dos modelos educativos: el tradicional, y el intercultural. El cuadro contiene resultados de manera concentrada del trabajo realizado con cuatro grupos focales.

Cuadro 2. Diferencias entre educación tradicional e interculturalidad

\begin{tabular}{|l|l|}
\hline \multicolumn{1}{|c|}{ Educación tradicional } & \multicolumn{1}{c|}{ Educación intercultural } \\
\hline - Maestro máxima autoridad poseedor de todos & - Una cultura respeta y convive con varias \\
los conocimientos, no propicia la reflexión ni el & $\begin{array}{l}\text { culturas, reconoce cada una sin menosprecio } \\
\text { de ninguna }\end{array}$ \\
análisis en los estudiantes & - Consideración de la lengua, la cultura, los \\
- Desconsideración de los conocimientos previos & valores, la cosmovisión, conocimientos \\
de los alumnos & $\begin{array}{l}\text { previos, intereses } \\
\text {-Uso y creación de materiales didácticos con } \\
\text { - Utilización de materiales descontextualizados }\end{array}$ \\
& $\begin{array}{l}\text { los recursos naturales con los que cuenta el } \\
\text { niño }\end{array}$ \\
\hline
\end{tabular}




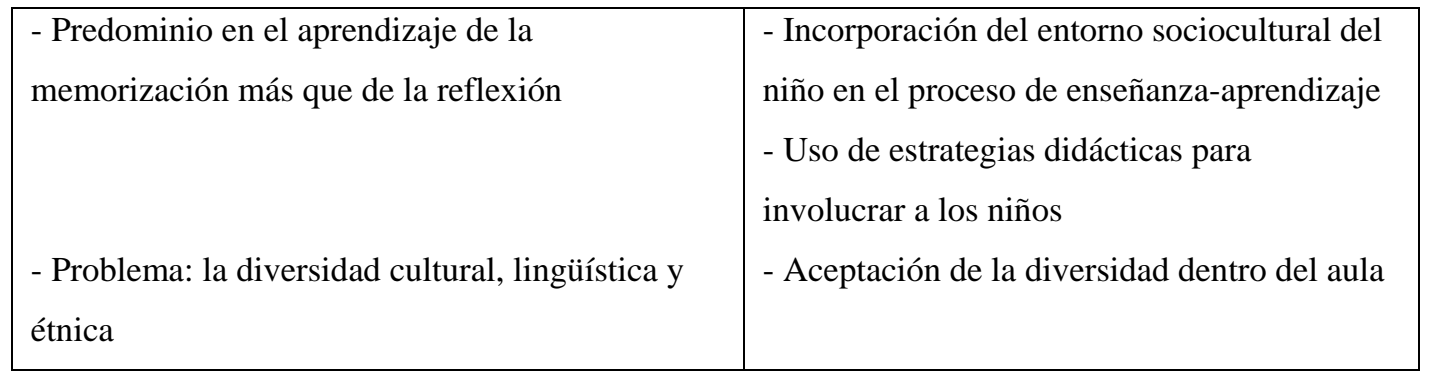

Fuente: elaboración a partir del trabajo con grupos focales de estudiantes, ENIIB.

Del cuadro anterior llama la atención que se establecen claramente las diferencias entre la educación tradicional y la intercultural. Con referencia a la educación tradicional, la podemos relacionar con lo que Freire (1990) denomina «educación bancaria», es decir: «el educador es el sujeto del proceso y el educando su objeto».

Por otra parte, en lo referente a la educación intercultural se puede observar que los estudiantes reconocen características importantes de este paradigma, como por ejemplo: aceptación de la diversidad, una educación adecuada al contexto sociocultural del estudiante donde él tiene un papel fundamental para la construcción del conocimiento.

Sin embargo, llama la atención que la base sobre la cual se representa la educación intercultural es el respeto y la convivencia entre varias culturas. Esta tendencia de considerar la interculturalidad más como una herramienta para favorecer la solidaridad entre las culturas, tiene que ver con el enfoque implementado en Europa a partir de las migraciones de africanos, latinos, orientales y europeos del este a los países con mejores condiciones de desarrollo; posteriormente fue exportado a América Latina. Este enfoque más que intercultural es claramente multicultural, ya que prioriza la convivencia entre las culturas antes que modificar las formas injustas en que se dan las relaciones.

A pesar de ello, es importante recalcar la claridad conceptual para diferenciar significativamente los dos tipos de educación. No obstante, cuando se les preguntó qué tipo de educación les impartían, es decir, si realmente llevaban una educación intercultural o si 
más bien es lo que se esperaba que hicieran cuando egresaran y estuvieran trabajando en las comunidades. Se mencionó que «...es algo muy complejo que difícilmente lo socializamos, y por ejemplo acá a través de nuestra experiencia en el salón de clases hablamos cuatro lenguas, y yo quisiera ver si realmente hay una aceptación, algún respeto, alguna integración, quisiera, no sé, preocuparme por aprender eso, pero no se da» (estudiante en grupo focal). «...aquí lo necesario es que ya se ponga en práctica, y que se vaya aprender, aquí nos dan teoría, nos dan instrumentos y a veces se socializa, pero ya queda de nosotros aplicarlo en las prácticas que hacemos» (entrevista en grupo focal de estudiantes, ENIIB).

Lo expresado habla de que la educación intercultural como práctica aún está por implementarse, es decir, se pretende que lo practiquen los estudiantes en sus prácticas en las comunidades y posteriormente como egresados en su lugar de trabajo, mientras tanto dentro del aula en ENIIB se siguen implementando métodos educativos tradicionales.

\section{LA INTERCULTURALIDAD DENTRO Y FUERA DE LA NORMAL}

A partir del trabajo grupal y las entrevistas se analizó qué tanto la interculturalidad ha trascendido más allá del espacio educativo. Es decir, la forma en cómo se vive la interculturalidad dentro y fuera de ENIIB.

En Gráfica 2 se puede observar que hay una tendencia de los estudiantes hacia una valoración positiva (con más de 52.2\%), lo que la convierte en la más alta con respecto de las demás. Al reflexionar con los estudiantes sobre los resultados respondieron lo siguiente:

- "Yo diría que normalmente la interculturalidad no está tanto allá afuera porque los mestizos nos discriminan, cuando usamos la lengua, nos llaman indios".

- “Aquí estamos entre indígenas, nos sentimos entre hermanos, pero allá nos van a humillar, nos van a burlar los que nos escuchan, porque allá están los mestizos”.

- "Porque se cumple el objetivo de la escuela que es el respeto la lengua, la cultura, en cada lengua que pertenecemos nos presentamos ... tenemos pues esa cosa de aprender otra cultura y les preguntamos a los compañeros que saben otra lengua y aprendemos otras palabras". 
- "Yo me siento como si estuviera en mi casa, porque puedo hablar en tseltal como mis compañeros, puede decir lo que siento, lo que pienso, sobre todo hablar de mi pueblo" (entrevistas grupos focales estudiantes ENIIB).

Comentarios de este tipo fueron frecuentes al reflexionar sobre la interculturalidad en la Normal; en ellos se reconoce la importancia del espacio educativo para manifestar libremente su diversidad lingüística y cultural, y se incorporaran en mayor o menor medida algunos de los «elementos de la cultura» - analizados anteriormente-, que permiten la incorporación de elementos de identidad de su comunidad. Esta situación, mencionan los estudiantes no tenía cabida en niveles educativos previos a su ingreso en ENIIB, ni cuando volvían a sus contextos de vida cotidiana.

Hay que recordar que la educación indígena ha sido el punto central de diversas discusiones, se le ha considerado como una respuesta a las necesidades de reconocimiento de las comunidades étnicas, como parte de la multiculturalidad y plurilingüismo del país. En este sentido, ENIIB es la reivindicación de un espacio negado por décadas a los estudiantes indígenas. No obstante, es necesario ligar la educación al contexto inmediato, problematizando la realidad y proponiendo acciones inmediatas dirigidas a la sociedad mestiza, pues de lo contario estaremos hablando de una educación indígena bilingüe, pero no intercultural. $\mathrm{O}$ en todo caso, el significado otorgado a la interculturalidad queda restringido al resultado de las interacciones que puedan surgir de las culturas originarias presentes en la Escuela Normal Indígena Intercultural Bilingüe «Jacinto Canek». 
Gráfica 2. Interculturalidad dentro de la Normal

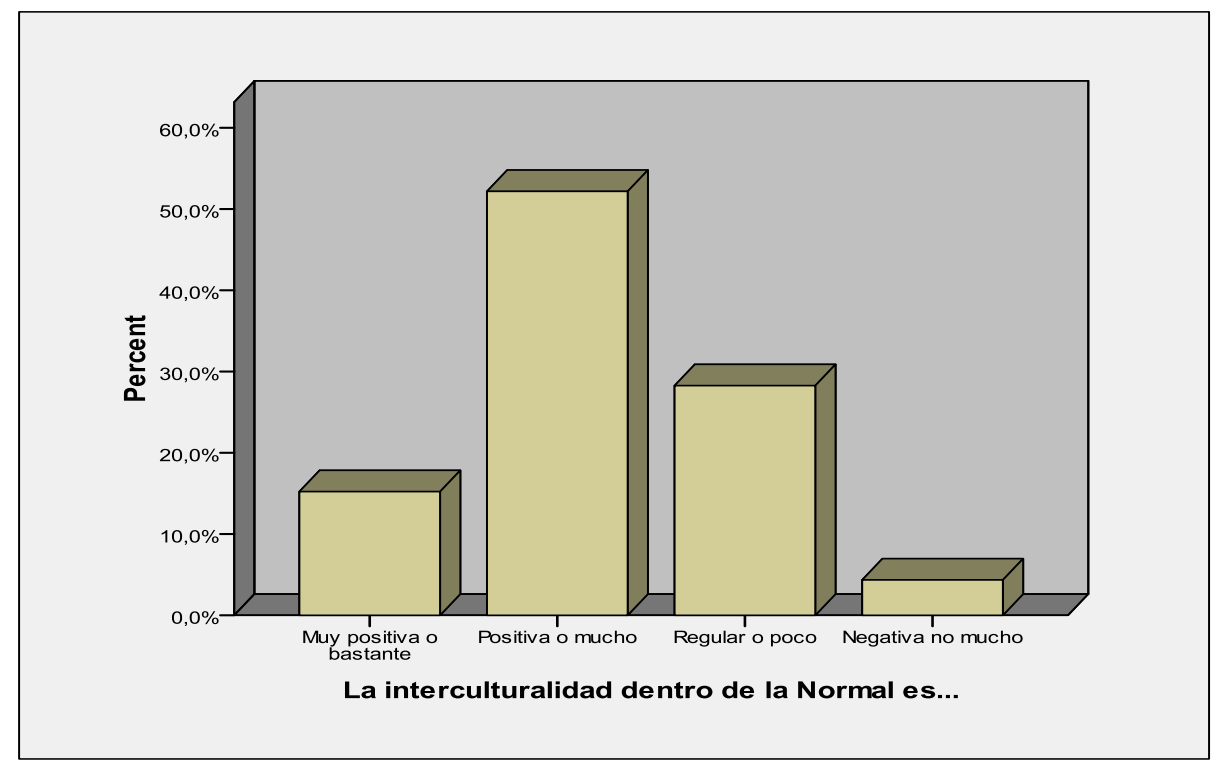

Fuente: Elaborado a partir de los resultados en grupo focal.

Lo anterior se contrapone a cuando se reflexionó con los estudiantes sobre la forma en cómo se vive la interculturalidad fuera de la Normal. Los resultados se pueden observar en la siguiente gráfica. 
Gráfica 3. Interculturalidad fuera de la Normal

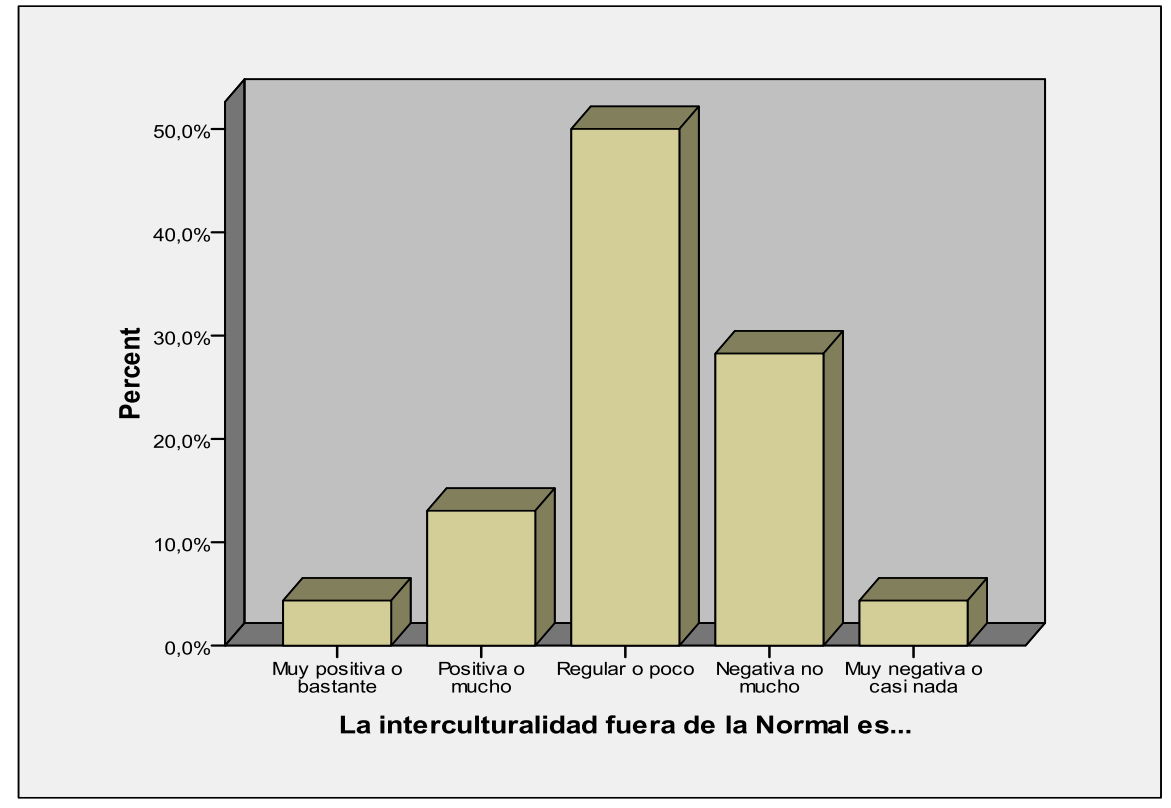

Fuente: Elaborado a partir de los resultados en grupo focal.

Como puede observarse, la «forma en que se vive la interculturalidad fuera de la ENIIB», es valorada como regular o poca (50\%), le sigue negativa o no mucho (30\%), esto se debe a que los estudiantes fuera de la escuela se enfrentan a otra realidad, dominada por formas de racismo silencioso y abierto que no permiten generar condiciones básicas para establecer relaciones de respeto y aprendizaje mutuo. En este sentido, se menciona que «...aquí se habla mucho de la reivindicación de la cultura, pero no se lleva a cabo cuando platicamos con otras personas, por ejemplo con los mestizos nos avergonzamos» (entrevista en grupo focal).

De esta manera, no basta tan solo que al interior de ENIIIB se genere un ambiente armónico entre las culturas, es necesario para la consolidación de la interculturalidad involucrar en el proceso de construcción al conjunto de la sociedad. Los estudiantes reconocen que a pesar de no sostener relaciones favorables para la interculturalidad fuera de la escuela - de manera particular con los mestizos-, en las comunidades sí 
interaccionan de manera diferente, y es que señalan que el enfoque intercultural les ha cambiado la forma en cómo se relacionan con las demás personas,

...creo que así directamente no hemos trabajado mucho así con gente grande de nuestra comunidad, pero porque no decir que cuando nosotros hemos ido a la observación y práctica, según la cultura de nuestras comunidades no debe de haber cercanía tanto niños como niñas, entonces se dice que si yo me agarro con una niña pues ya es mi novia, entonces se empieza a mal interpretar las cosas, por eso creo que con este nuevo enfoque estamos tratado de acercar a los niños a la integración a una educación diferente (entrevista en grupo focal, estudiantes ENIIB).

Es evidente que la interculturalidad fuera del espacio educativo de ENIIB no existe, no hay elementos que indiquen lo contrario, es decir, aún están presentes formas de racismo y discriminación abierta y silenciosa, la negación cultural y la inequidad entre las relaciones interculturales, circunstancias que son producto del sistema educativo y en general del desarrollo de la sociedad.

\section{COMENTARIOS FINALES}

Existe una necesidad por revalorar los elementos culturales de cada estudiante e incorporarlos en un proceso de formación que esté más relacionado con su contexto social y cultural. Con ello se comenzarán a trasformar las condiciones de desigualdad social en la que se encuentran las comunidades. En este sentido, la Escuela Normal «Jacinto Canek» propone un cambio radical en la educación para que en cierto sentido dé respuesta a las necesidades de nuestra sociedad multicultural.

Para lograr una educación en la cual se respeten las diferencias de cada individuo y se busque la equidad, se necesita más que la disposición de aprender del otro; se requiere de un cambio de actitud del sujeto que ayude a resignificar el actuar de la sociedad en su conjunto. Es decir, implica redefinir las formas de relación entre los sujetos y las culturas. En este sentido, por los resultados encontrados, se puede decir que hace falta mayor comprensión del paradigma intercultural y sobre su aplicación como propuesta pedagógica, 
no obstante existe un reconocimiento de la diversidad sociocultural con que cuentan los estudiantes y los incorporan en el proceso de enseñanza-aprendizaje. Sin embargo, es necesario realizar adecuaciones específicas a las estrategias pedagógicas con las que se forma a los estudiantes.

Sin duda uno de los mayores desafíos para la interculturalidad es comenzar a generar nuevas formas de relación entre las culturas basadas en una perspectiva de aprendizaje mutuo, respeto y aceptación del otro. A pesar de que en ENIIB se tienen elementos importantes para comprender la interculturalidad sus interpretaciones giran en torno a la multiculturalidad, por lo que aún falta consolidar una propuesta educativa intercultural para la Normal Indígena «Jacinto Canek».

La propuesta principal de ENIIB es que el enfoque de educación intercultural implique modificaciones en las prácticas educativas convencionalmente aplicadas en las comunidades; se plantea como una educación diferente para los pueblos indígenas y para dar una respuesta a los modelos educativos que no consideran los elementos culturales propios de cada región. Esto nos habla de la contradicción con el paradigma intercultural, ya que restringe su área de influencia solamente hacia las comunidades indígenas.

Como se ha visto, se busca que los estudiantes realicen los cambios significativos en la educación al momento de que realizan sus prácticas profesionales, como por ejemplo: que consideren la realidad sociocultural del contexto en que se encuentran los niños y niñas, que diseñen materiales didácticos de acuerdo con la región para facilitar el proceso educativo, que innoven las estrategias pedagógicas, que utilicen la lengua materna como puente principal para establecer la comunicación con los niños y que incorporen metodologías en el currículo para reconstruir el espacio de aprendizaje en donde se involucran saberes, conocimientos y valores tradicionales. 
Los estudiantes de alguna manera consideran los elementos antes mencionados para generar cambios significativos en la educación de las comunidades indígenas. Sin embargo, es necesario considerar tanto los conocimientos de la cultura propia así como también de la cultura universal, y a partir de la reflexión y análisis proponer nuevas y variadas formas de aprender.

De acuerdo con los resultados se observa un significativo avance en la discusión teórica entre los maestros y estudiantes sobre cómo debe llevarse a cabo una educación intercultural en contextos multiculturales; sin embargo, no se aplica en ENIIB por la presencia de elementos característicos de una «educación tradicional», lo que hasta cierto punto es contradictorio con una propuesta educativa intercultural. Aún falta trascender hacia una práctica educativa intercultural.

Finalmente, no debemos olvidar que el significado otorgado a la educación intercultural depende del contexto en el que se desarrolle y de los elementos culturales con los que se cuente. Es por ello que el significado que se le otorga a la interculturalidad en ENIIB se deriva a partir de la armonía entre los grupos hablantes de una lengua materna. De ahí que en su construcción se retomen como principales elementos la cuestión indígena y el bilingüismo. 


\section{BIBLIOGRAFÍA}

Barth, F. (comp.), 1976, Los grupos étnicos y sus fronteras, FCE, México.

Beltrán, J., 2003, «El profesor del siglo XXI», Segundo Congreso Internacional de Educación en Chiapas: Sus tiempos y espacios, Universidad Autónoma de Chiapas, México.

Bertely, M., 1998, «Educación indígena del Siglo XXI», en Un siglo de educación en México, Tomo II, Pablo Latapí, Fondo de Cultura Económica, México.

Blumer, H., 1986, «Symbolic Interactionism. Perspectives and Method», University of California, California.

Convenio 169 de la Organización Internacional del Trabajo. Página electrónica: http://www.cdi.gob.mx/transparencia/convenio169_oit.pdf

Díaz-Couder, E., 1998, «Diversidad Cultural y Educación en Iberoamérica», Revista Iberoamericana de Educación, n. 17, mayo-agosto.

Fornet, R., 2002, «Lo intercultural: el problema de su definición», en Intercultural el Balance y perspectivas, editado por Y. Onghena, Fundación CIDOB, Barcelona, España.

Freire, P., 1990, Pedagogía del oprimido, Siglo XXI, México.

Gashé, J., 2002, «El difícil reto de una educación indígena amazónica: alcances y abandonos», en Interculturalidad, sociedad multicultural y Educación Intercultural, Asociación alemana para la educación de adultos, CEAL, México. 
Giménez, G., 2002, «Paradigmas de identidad», en Sociología de la identidad, coordinado por A. Chihu, UAM, Porrúa, México.

Glaser, B., y A. Strauss, 1967, «The Discovery of Grounded Theory. Strategies for Qualitative Research. Aldine De Gruyter: Nueva York», citado en Wiesenfeld, E., 2001, La autoconstrucción, un estudio psicosocial del significado de la vivienda, Ed. Universidad Central de Venezuela, Facultad de Humanidades y Educación, y Comisión de Estudios de Postgrado-CEP, Venezuela.

Guba y Lincoln, 2000, «Paradigmas en competencia en la investigación cualitativa», en Por los Rincones. Antología de Métodos cualitativos en la investigación social, compilado por Denman y Haro, El Colegio de Sonora, Sonora.

Gutiérrez, R., 2006, «Impactos del zapatismo en la escuela: Análisis de la dinámica educativa indígena en Chiapas (1994-2004)», Revista Liminar, estudios sociales y humanísticos, año/v. IV, n. 1, junio, Universidad de Ciencias y Artes de Chiapas, México.

Hammersley, M. y Atkinson, P., 1994, Etnografía. Métodos de Investigación, Paidós, España.

López, L. y Küper, W., 1999, «La educación Intercultural Bilingüe en América Latina. Balances y perspectivas», Revista Iberoamericana de Educación, n. 20, mayo-agosto.

Mucchielli, A., 1996, «Diccionario de métodos cualitativos en ciencias humanas y sociales», Editorial Síntesis, Madrid.

Rodrigo, M., 1999, «Elementos para una comunicación intercultural», MINEDUC, España. 
Saldívar, A., 2003, «Interculturalidad, constructivismo y educación superior. Hacia la definición de estrategias educativas para la formación de indígenas en las universidades», en el foro La Interculturalidad en el desarrollo y la educación superior, Ecosur, UNACH, UPN, Universidad de Chapingo, México.

Saldívar, A. y S. Navarro, 2007, «Retos para la formación superior de estudiantes indígenas en Chiapas. Informe Técnico». FAEIB, Innovación y apoyo educativo, A. C., México.

Schmelckes, S., 2003, Interculturalidad y educación, SEP, México.

2001, «La combinación de estrategias cuantitativas y cuantitativas en la investigación educativa: reflexiones a partir de tres estudios», Revista Mexicana de Investigación Educativa, COMIE, México.

Secretaría de Educación Pública-Poder Ejecutivo Federal, 1996, «Programa de desarrollo educativo 1995-2000», SEP, México.

Secretaría de Educación Pública-Coordinación General de Educación Intercultural Bilingüe, 2004, Imágenes de un andar, SEP-CGEIB, México.

Stavenhagen, R., 2000, «México: minorías étnicas y política cultural», en Antología Temática Ética y Calidad en la educación, UPN, México.

UNESCO, 2001, «Declaración Universal de la UNESCO sobre la diversidad Cultural», en http://www.campus-e.org/pensariberoamerica/ric01documentos02.html

Wiesenfeld, E., 2001, «La autoconstrucción, un estudio psicosocial del significado de la vivienda», Ed. Universidad Central de Venezuela, Facultad de Humanidades y Educación y Comisión de Estudios de Postgrado-CEP, Venezuela. 
Zemelman, H. (coord.), 1990, Cultura y política en América Latina, Siglo XXI, México.

\section{Notas}

\footnotetext{
${ }^{1}$ Actualmente la escuela se ubica en el municipio de Zinacantán, a 15 minutos de San Cristóbal de Las Casas. El estudio se realizó durante 2007 cuando se ubicaba en instalaciones provisionales de la Escuela Primaria «Jean Piaget» en las inmediaciones de la ciudad.

${ }^{2}$ Las escuelas autónomas zapatistas incluyen actualmente desde primaria y secundaria hasta universidad, como la denominada Universidad de la Tierra que opera actualmente en San Cristóbal de Las Casas. Otra experiencia de escuelas autónomas es la propuesta de Universidad de la Montaña promovida por los maristas, que se está implementando en la zona de Las Cañadas, municipio Las Margaritas y La Independencia, en la Selva Lacandona.

${ }^{3}$ Jacinto Canek, (1730-1761) representa la figura del jefe rebelde, caudillo y mártir de la lucha anticolonial de los mayas de Yucatán. Formado por los franciscanos, se proclama rey maya, y junto a cientos de seguidores sensibles a su discurso mesiánico, lanza una insurrección (que fue reprimida) en contra de las autoridades novohispanas exigiendo la abolición del tributo. Es así que el haberle otorgado el nombre de Jacinto Canek a ENIIB significa una constante lucha en contra de un sistema educativo excluyente.

${ }^{4}$ Esta colonia se encuentra en las inmediaciones de San Cristóbal de Las Casas habitada principalmente por desplazados de comunidades circunvecinas a causa de problemas de índole religioso y político.

${ }^{5}$ Financiado por el Fondo de Apoyo a la Educación Indígena Intercultural, FONDEIB, en 2002.

${ }^{6}$ En San Cristóbal de Las Casas, Chiapas, opera el Campus III de la UNACH (Facultad de Derecho, Facultad de Ciencias Sociales, Escuela de Lenguas), la Universidad Intercultural de Chiapas, la Escuela Normal Indígena Intercultural Bilingüe «Jacinto Canek», la Normal Superior «Fray Matías de Córdova y Ordoñez» y la Normal Superior "Manuel Larráinzar". Además de las universidades privadas (tres).

${ }^{7}$ Se trabajó con cuatro grupos focales, al interior de cada grupo se pidió que hicieran una caracterización de los dos tipos de educación, posteriormente se discutió en plenaria los resultados.
}

Fecha de recepción: 23 de septiembre de 2010.

Fecha de aceptación: 25 de febrero de 2011. 